Supporting Information

\title{
Co-N Decorated Hierarchically Porous Graphene Aerogel for Efficient Oxygen Reduction Reaction in Acid
}

Xiaogang Fu, Ja-Yeon Choi, Pouyan Zamani, Gaopeng Jiang, Md. Ariful Hoque Fathy Mohamed Hassan and Zhongwei Chen*

Department of Chemical Engineering, Waterloo Institute for Nanotechnology, Waterloo Institute of Sustainable Energy, University of Waterloo, 200 University Ave. W, Waterloo, ON, N2L 3G1, Canada

E-mail: zhwchen@uwaterloo.ca 


\section{Experimental Section}

\section{Chemicals and materials}

Graphite powder (microcrystal grade, APS 2-15 micron, 99.9995\%) was purchased from (Alfa Aesar). Nafion (5 wt\% solution in a mixture of lower aliphatic alcohols and water) was purchased from Aldrich. The other chemicals such as aniline, $\mathrm{Co}(\mathrm{NO} 3)_{2} \cdot 6 \mathrm{H}_{2} \mathrm{O}, \mathrm{KClO}_{4}, \mathrm{H}_{2} \mathrm{SO}_{4}, \mathrm{H}_{3} \mathrm{PO}_{4}, \mathrm{KMnO}_{4}$ and $\mathrm{H}_{2} \mathrm{O}_{2}$ were purchased from Aldrich.

\section{Synthesis of Graphene Oxide (GO):}

GO was prepared from graphite powder using an improved method reported previously by Marcano et al. ${ }^{1}$ Briefly, $2 \mathrm{~g}$ of graphite powder was added to $400 \mathrm{~mL}$ of a 9:1 volumetric mixture of concentrated $\mathrm{H}_{2} \mathrm{SO}_{4} / \mathrm{H}_{3} \mathrm{PO}_{4}$ in an ice bath. $18 \mathrm{~g}$ of $\mathrm{KMnO} 4$ was then added slowly and the reaction was heated to $50{ }^{\circ} \mathrm{C}$ and held for 12 hours. Deionized (DI) water $(400 \mathrm{~mL})$ then was slowly added to the mixture. Finally $15 \mathrm{~mL}$ of $\mathrm{H}_{2} \mathrm{O}_{2}(30 \%)$ was added in order to complete the oxidation reaction, and the final mixture was centrifuged to separate out the solids which were washed thoroughly with water, ethanol and $\mathrm{HCl}(30 \%)$; followed by lyophilization.

\section{Synthesis of PANI:}

$2.0 \mathrm{~mL}$ of aniline was dispersed in $300 \mathrm{~mL}$ of $0.5 \mathrm{M} \mathrm{HCl}$ solution. The solution was kept below $10^{\circ} \mathrm{C}$ while $3 \mathrm{~g}$ of ammonium peroxydisulfate was added slowly. After constant stirring for 24 hours in dark place, aniline was completely polymerized to polyaniline (PANI). Filter and wash the samples with DI water three times and dry in a vacuum oven at $80^{\circ} \mathrm{C}$ for overnight to obtain the PANI powder. ${ }^{2}$ 


\section{Physical Characterization:}

Surface morphologies of samples were investigated using SEM (LEO FESEM 1530) and TEM (TEM, JEOL 2010F). X-ray diffraction (XRD) measurements were recorded with Bunker AXS D8 Advance. Surface area and pore size were determined by a surface area and porosity analyzer (Micromeritics Instrument Corp. ASAP2020). XPS spectra were collected using a Thermal Scientific K-Alpha XPS spectrometer. Raman spectroscopy was carried out on a Bruker Senterra Raman Microscope operating with a wavelength of $532 \mathrm{~nm}$.

Electrochemical experiments were conducted on a workstation (Pine Instrument Co., AFCBP-1) assembled with a rotation speed controller (Pine Instrument Co., AFMSRCE) using a conventional three compartment electrochemical cell. $\mathrm{Ag} / \mathrm{AgCl}$ electrode $(3 \mathrm{M} \mathrm{KCl})$ and graphitic electrode were used as reference and counter electrode, respectively. All potentials in this study were converted to RHE scale. A rotating ring-disk electrode (RRDE) with a Pt ring (5.52 mm inner-diameter and 7.16 $\mathrm{mm}$ outer-diameter) served as the working electrode. The catalyst ink was prepared by dispersion the catalyst powder $(10 \mathrm{mg})$ with $1 \mathrm{~mL}$ 1-Propanol in an ultrasonic bath for $2 \mathrm{~h}$. Then $12 \mu \mathrm{L}$ of the catalyst ink was pipetted onto the glass carbon surface and dried thoroughly in air, after that $3 \mu \mathrm{L}$ of $0.05 \mathrm{wt} \%$ Nafion solution was dropped onto the catalyst layer, leading to a catalyst loading of approximately $0.6 \mathrm{mg} \mathrm{cm}^{-2}$. The ORR activities were measured in $\mathrm{O}_{2}$ saturated $0.5 \mathrm{M} \mathrm{H}_{2} \mathrm{SO}_{4}$ and $0.1 \mathrm{M} \mathrm{KOH}$ from 1.0 $\mathrm{V}$ to $0.0 \mathrm{~V}$ with a scan rate of $10 \mathrm{mV} \mathrm{s}^{-1}$. For detecting $\mathrm{H}_{2} \mathrm{O}_{2}$ formed at the disc electrode, the ring potential in the RRDE system was set to $1.20 \mathrm{~V}$ ( $v s$ RHE). Commercial Pt/C (TKK, 28.2 wt. \% Pt) with a Pt loading of $20 \mu \mathrm{g} \mathrm{cm}^{-2}$ was measured in $0.1 \mathrm{M} \mathrm{HClO}_{4}$ and $0.1 \mathrm{M} \mathrm{KOH}$ electrolyte for comparison. Accelerated degradation testing (ADT) was carried out by cycling the electrode potential 5000 times under nitrogen saturated electrolyte between $0.6 \mathrm{~V}$ and $1.0 \mathrm{~V} v s$ RHE at a scan rate of 50 $\mathrm{mV} \mathrm{S}^{-1}$. For the $\mathrm{MeOH}$ resistance testing, $1 \mathrm{M} \mathrm{MeOH}$ solution was introduced into the oxygen saturated electrolyte to examine the methanol crossover affection. 
The four-electron selectivity of catalysts was evaluated based on the $\mathrm{H}_{2} \mathrm{O}_{2}$ yield, calculated from the following equation: ${ }^{3}$

$$
\begin{aligned}
& n=4 \times \frac{I_{D}}{I_{D}+I_{R} / N} \\
& H_{2} \mathrm{O}_{2} \%=200 \times \frac{I_{R} / N}{I_{R} / N+I_{D}}
\end{aligned}
$$

Here, $I_{D}$ and $I_{R}$ are the disk and ring currents, respectively, and $N=0.26$ is the ring collection efficiency 

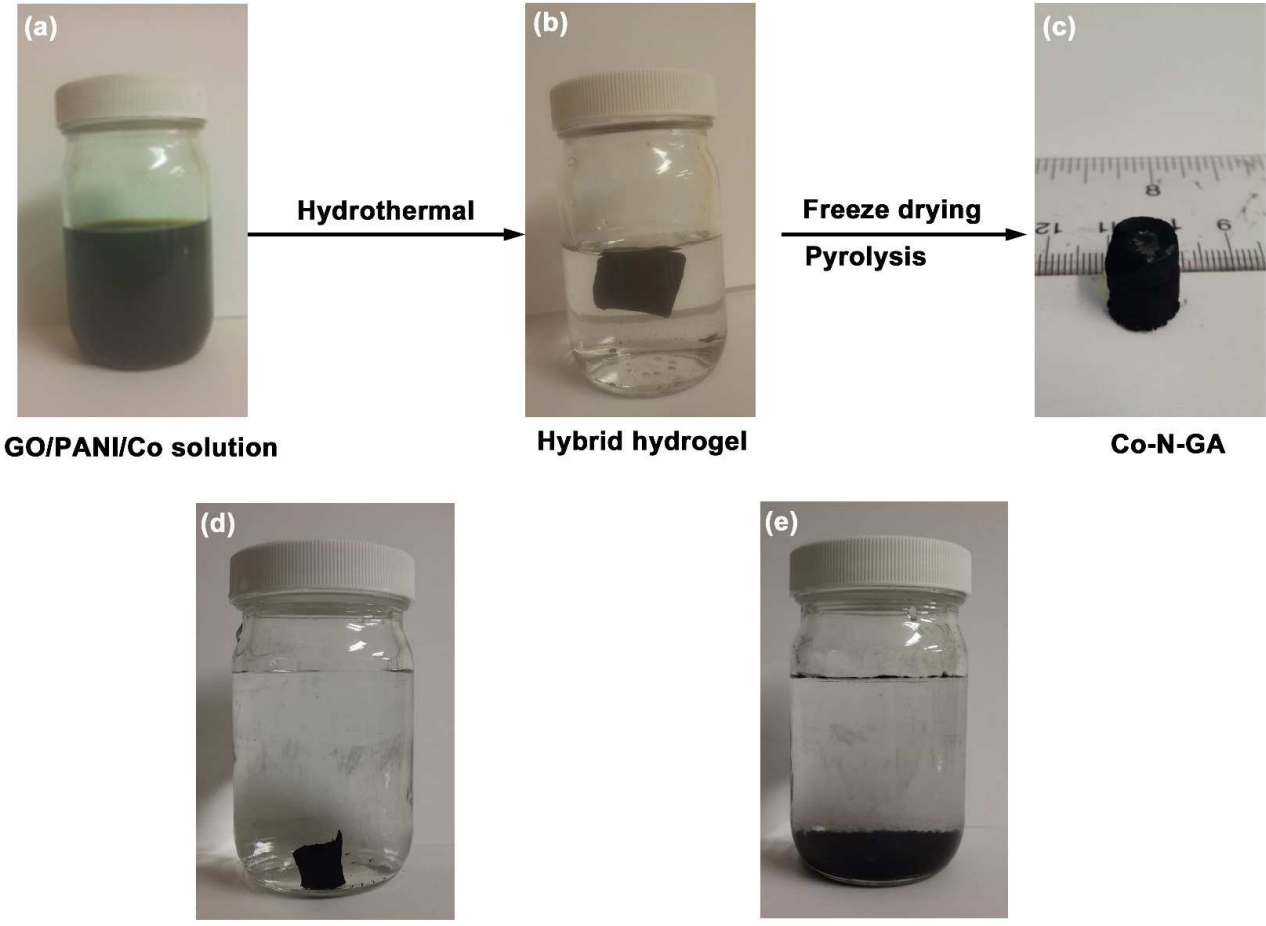

GO hydrogel without PANI

GO precipitation

Figure S1. (a), (b) and (c): fabrication process for the porous Co-N-GA catalyst; (d): the GO hydrogel prepared in the absence of PANI and cobalt salt; (e): GO based precipitation. 

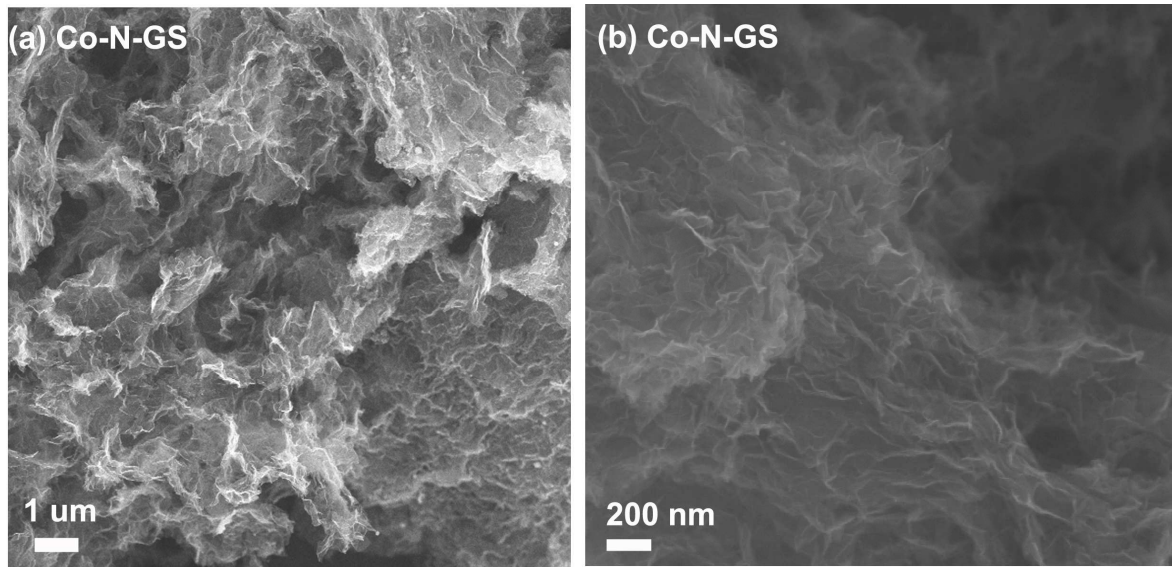

Figure S2. (a), (b) SEM images of Co-N-GS 

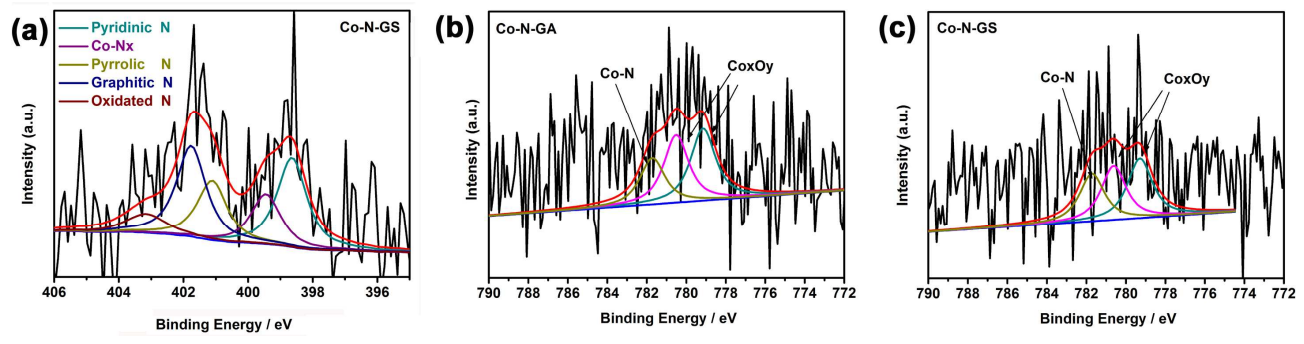

Figure S3. High resolution N 1s peak of the (a) Co-N-GS; (b) and (c): High resolution Co $2 p$ peak of the Co-N-GA and Co-N-GS. 


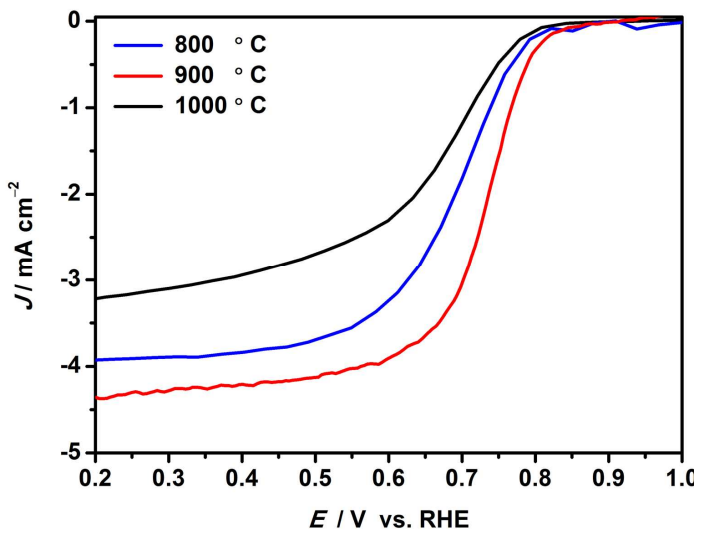

Figure S4. Polarization curves of the Co-N-GA catalysts pyrolyzed at $800{ }^{\circ} \mathrm{C}, 900{ }^{\circ} \mathrm{C}$ and $1000{ }^{\circ} \mathrm{C}$. Test conditions: $0.5 \mathrm{M} \mathrm{H}_{2} \mathrm{SO}_{4}$ solution, $10 \mathrm{mV} \mathrm{s}^{-1}$ scan rate and $900 \mathrm{rpm}$ rotate rate. 

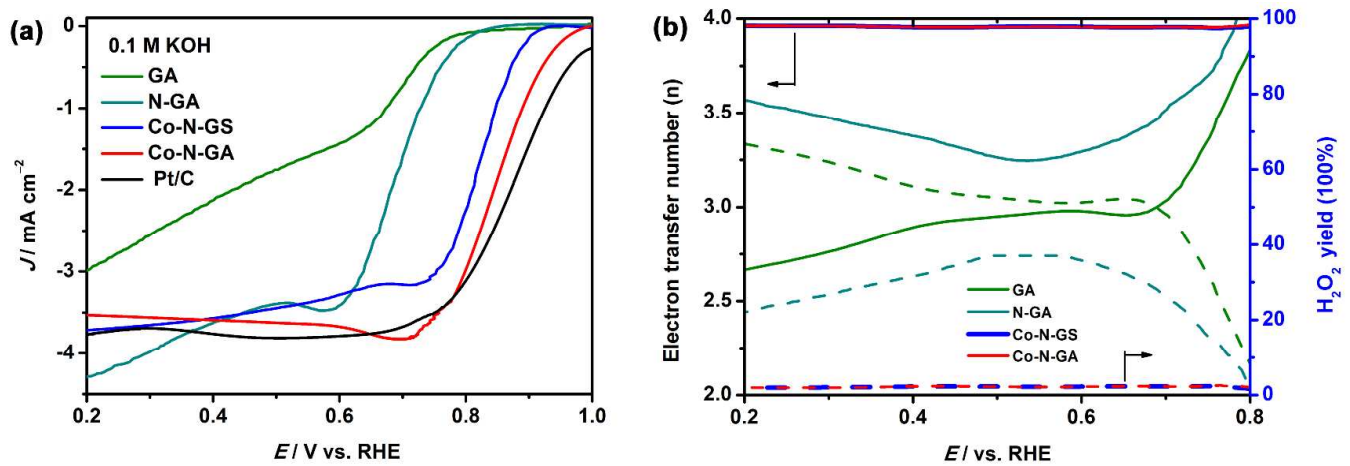

Figure S5. (a) RRDE polarization curves of the GA, N-GA, Co-N-GS, Co-N-GA and $\mathrm{Pt} / \mathrm{C}$ in $0.1 \mathrm{M} \mathrm{KOH}$ at a scan rate of $10 \mathrm{mV} \mathrm{s}^{-1}$ and $900 \mathrm{rpm}$; (b) electron-transfer number and $\mathrm{H}_{2} \mathrm{O}_{2}$ yield of the catalysts. 
Table S1. Elemental composition of the samples obtained from XPS results.

\begin{tabular}{lllll}
\hline Samples & C (at.\%) & N (at.\%) & O (at.\%) & Co (at.\%) \\
\hline N-GA & 93.80 & 2.86 & 3.34 & --- \\
Co-N-GS & 94.63 & 2.57 & 2.52 & 0.29 \\
Co-N-GA & 94.54 & 2.10 & 3.12 & 0.24 \\
\hline
\end{tabular}


Table S2. Atomic concentrations (at.\%) of heterocyclic N components of samples in the $\mathrm{N} 1 \mathrm{~s}$ binding energy region $(398 \sim 405 \mathrm{eV})$.

\begin{tabular}{lcllll}
\hline & Pyridinic N & Co-N & Pyrrolic N & Graphitic N & Oxidated N \\
& $\sim 398 \mathrm{eV}$ & $\sim 399 \mathrm{eV}$ & $\sim 400 \mathrm{eV}$ & $\sim 401 \mathrm{eV}$ & $\sim 402-404 \mathrm{eV}$ \\
\hline N-GA & $43.75 \%$ & --- & $25.2 \%$ & $24.5 \%$ & $6.5 \%$ \\
Co-N-GS & $26.3 \%$ & $17.9 \%$ & $18.9 \%$ & $28.6 \%$ & $8.2 \%$ \\
Co-N-GA & $25.8 \%$ & $18.7 \%$ & $21.4 \%$ & $29.8 \%$ & $4.2 \%$ \\
\hline
\end{tabular}

1. Marcano, D. C.; Kosynkin, D. V.; Berlin, J. M.; Sinitskii, A.; Sun, Z.; Slesarev, A.; Alemany, L. B.; Lu, W.; Tour, J. M., Improved Synthesis of Graphene Oxide. ACS Nano 2010, 4, 4806-4814.

2. Rahy, A.; Sakrout, M.; Manohar, S.; Cho, S. J.; Ferraris, J.; Yang, D. J., Polyaniline Nanofiber Synthesis by Co-Use of Ammonium Peroxydisulfate and Sodium Hypochlorite. Chem. Mater. 2008, 20, 4808-4814.

3. Gojković, S. L.; Gupta, S.; Savinell, R. F., Heat-Treated Iron(III) Tetramethoxyphenyl Porphyrin Chloride Supported on High-Area Carbon as An Electrocatalyst for Oxygen Reduction: Part III. Detection of Hydrogen-Peroxide During Oxygen Reduction. Electrochim. Acta 1999, 45, 889-897. 